\title{
МЕТОД ОПРЕДЕЛЕНИЯ ДВИЖУЩЕЙ СИЛЫ ПРОЦЕССА РАСТЕКАНИЯ ЖИДКОСТИ ПО ПОВЕРХНОСТИ ТВЕРДОГО ТЕЛА
}

Растекание жидкостей по поверхности твердых тел играет важную роль во многих физико-химических явлениях и технологических процессах. В связи с этим в последнее время значительное внимание уделяют исследованию кинетики растекания в различных системах; особенно подробно изучена кинетика смачивания жидкими металлами.

Растекание термодинамически возможно при условии $K \sigma_{23}>K \sigma_{13}+\sigma_{12}$, где $K-$ коэффициент шероховатости твердой поверхности [ $\left.{ }^{1}\right], \sigma_{23}, \sigma_{13}$, $\sigma_{12}$ - значения удельной свободной поверхностной энергии на границах раздела твердое тело-окружающая среда, твердое тело-жидкость, жидкость - среда. Из количественной теории растекания $\left[{ }^{2,3}\right]$ следует, что произведение убыли удельной свободной поверхностной энергии в процессе смачивания $\Delta \sigma$ на длину периметра смачивания $\Delta \sigma a=\left(K \sigma_{23}-\right.$ $\left.-K \sigma_{13}-\sigma_{12}\right) a$ численно равно движущей силе процесса течения смачивающей жидкости по поверхности твердого тела (при отсутствии других внешних сил, способствующих течению). От величины $\Delta \sigma$ зависят такие важнейшие характеристики растекания, как скорость процесса и размеры площади, которую может смочить капля заданного объема. Таким образом, знание величины $\Delta \sigma$ принципиально необходимо для количественного описания процесса растекания 'и различных прикладных задач, например, для оценки некоторых свойств припоев [ [4].

В большинстве случаев прямой расчет $\Delta \sigma$ невозможен, так как значения $\sigma_{13}$ и $\sigma_{23}$ обычно неизвестны. В связи с этим возникает необходимость в разработке косвенных методов оценки $\Delta \sigma$. В работе [5] предложен способ, основанный на измерении высоты подъема жидкости вдоль вертикально расположенной поверхности твердого тела. Окончанию подъема соответствует условие $\Delta \sigma=\varrho g x_{0} \delta$. Здесь $\mathrm{Q}-$ плотность жидкости, $\delta-$ толщина поднимающегося слоя жидкости, $g$ - ускорение силы тяжести, $x_{0}$ - конечная высота подъема. Непосредственное измерение толщины слоя непрозрачных жидкостей затруднено. В таких случаях величину $\delta$ можно определить исходя из теоретической и экспериментальной зависимостей $x=x(t) \quad(x-$ высота подъема, $t-$ время $)$. Основная цель данной работы состоит в количественном описании кинетики подъема смачивающей жидкости по наклонной поверхности твердого тела и развитии метода определения величины убыли удельной свободной поверхностной энергии в процессе растекания.

Рассмотрим подъем слоя смачивающей ньютоно́вской жидкости постоянной толщины $\delta \ll x$ по наклонной пластине периметра $a$ от источника массой $M \gg m(m-$ масса поднимающегося слоя жидкости) (рисунок). На единицу объема слоя жидкости действуют две внешние силы: 
движущая сила растекания $\frac{1}{\varrho} f_{S}=\frac{\Delta \sigma a}{m}\left[{ }^{2}\right]$ и сила тяжести $\frac{1}{\varrho} f_{g}=g \sin \alpha \quad(\alpha-$ угол наклона пластины, причем $\left.0^{\circ}<\alpha \leqslant 90^{\circ}\right)$. Если выполняются условия квазистационарного и безынерционного течения $\left[{ }^{2}\right]$, систему уравнений движения слоя можно свести к следующему выражению:

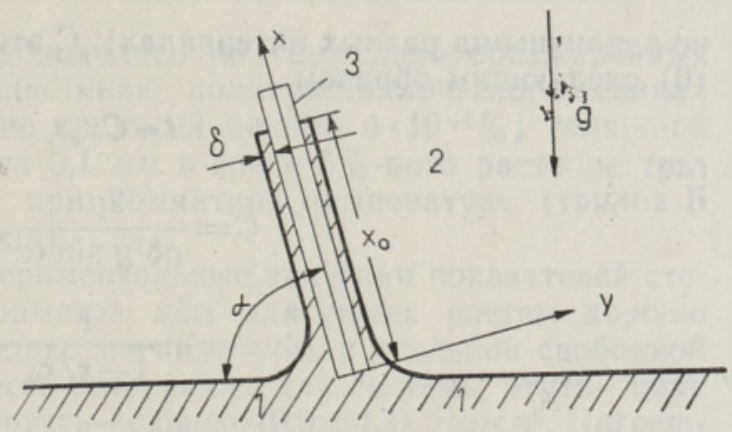

Подъем смачивающей жидкости по наклонной поверхности твердого тела: 1 - жидкость, 2 - среда, 3 - твердое тело.

$$
\frac{\mu}{\varrho} \frac{\partial^{2} u}{\partial y^{2}}+\frac{\Delta \sigma a}{m}-g \sin \alpha=0
$$

где $u$ - компонента скорости течения жидкости, направленная вдоль поверхности пластины, $\mu-$ вязкость жидкости.

Граничные условия на свободной поверхности жидкости

$$
\frac{\partial u}{\partial y}=0 \quad \text { при } \quad y=\delta
$$

и на границе жидкость-твердое тело

$$
u=0 \text { при } y=0 .
$$

В случаях, не осложенных химическими реакциями, интенсивным массообменом и т. д., можно принять, что $\mu$ и $\Delta \sigma$ постоянны. Тогда из (1), с учетом граничных условий (2) и (3), получим выражение для скорости передвижения фронта жидкости

$$
\frac{d x}{d t}=\frac{\varrho}{2 \mu}\left(\frac{\Delta \sigma \alpha}{m}-g \sin \alpha\right) \delta^{2} .
$$

Учтя начальное условие

$$
x=0 \text { при } t=0 \text {, }
$$

получим соотношение между высотой и временем подъема

$$
t=\frac{2 \mu x_{0}}{\varrho \delta^{2} g \sin \alpha}\left[\ln \frac{x_{0}}{x_{0}-x}-\frac{x}{x_{0}}\right] .
$$

Здесь $x_{0}=\frac{\Delta \sigma}{\operatorname{\varrho g} \delta \sin \alpha}-$ конечная высота поднятия.

Непосредственный расчет толщины пленки жидкости из соотношения (6) громоздок и трудоемок. Для облегчения расчета разобъем высоту подъема $x_{0}$ на несколько интервалов (количество интервалов определяется требуемой точностью расчета). В пределах каждого интервала заменим точное решение уравнения (6) приближенным соотношением вида $x=A t^{n}$ (значения $A$ и $n$ постоянны в пределах каждого интервала, 
но различны на разных интервалах). С этой целью запишем соотношение

(6) следующим образом

где

$$
t=C x_{0} z
$$

$$
\begin{aligned}
& C=\frac{2 \mu}{\varrho \delta^{2} g \sin \alpha}, \\
& z=\ln \frac{1}{1-x / x_{0}}-\frac{x}{x_{0}} .
\end{aligned}
$$

Выражение (9) можно приближенно заменить соотношением вида $z=B\left(x / x_{0}\right)^{m}$. Тогда задавшись погрешностями коэффициента пропорциональности $B$ и показателя степени $m(m=1 / n)$, методом наименьших квадратов соответствующие значения $B$ и $m$ можно рассчитать на каждом из интервалов. Такие расчеты были проведены на ЭВМ «Минск-22» при

\begin{tabular}{|c|c|c|c|c|c|c|c|}
\hline \multirow{2}{*}{\multicolumn{3}{|c|}{ Расчет }} & \multicolumn{5}{|c|}{ Эксперимент } \\
\hline & & & \multicolumn{2}{|c|}{ Ртуть-цинк } & \multicolumn{2}{|c|}{ 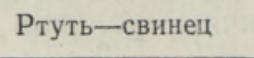 } & \multirow{2}{*}{$\Delta A / A$} \\
\hline$x / x_{0}$ & $B$ & $n$ & $n$ & $\underset{{ }_{9 p 2 / C M^{2}}}{\Delta \sigma,}$ & $n$ & $\underset{\Im p 2 / C M^{2}}{\Delta \sigma,}$ & \\
\hline $0,05-0,40$ & 0,83 & 0,47 & 0,46 & 4,3 & 0,49 & 1,0 & 0,3 \\
\hline $0,40-0,60$ & 1,47 & 0,35 & 0,34 & 3,9 & 0,34 & 0,8 & 0,2 \\
\hline $0,60-0,90$ & 2,15 & 0,25 & 0,23 & 3,9 & 0,23 & 0,8 & 0,1 \\
\hline
\end{tabular}
абсолютных погрешностях $\Delta B=0,04$ и $\Delta m=0,03$. Полученные результаты приведены в таблице.

Таким образом, кинетику подъема смачивающей жидкости можно с заданной степенью точности аппроксимировать следующими степенными соотношениями:

$$
x=\left(\frac{x_{0}^{m-1}}{C B}\right)^{\frac{1}{m}} t^{\frac{1}{m}}=A t^{n}
$$

Выражение вида (10) позволяет легко найти значение толщины слоя $\delta$. Для этого достаточно построить в двойном логарифмическом масштабе зависимость $x=x(t)$ и подставить найденное значение $A$ в формулу для толщины слоя

$$
\delta=\sqrt{\frac{2 \mu A^{m} B}{\varrho g x_{0}^{m-1} \sin \alpha}}
$$

Вместе с тем, разбив высоту подъема на несколько интервалов, можно в пределах каждого интервала независимо оценить значения $\delta$ и $\Delta \sigma$, что существенно повышает точность и надежность этих оценок. Проведенный расчет позволяет определить величины $\delta$ и $\Delta \sigma$ также в тех случаях, когда кинетика подъема жидкости известна только на одном из интервалов высоты подъема.

В качестве примера приведем расчет убыли удельной свободной поверхностной энергии в процессе растекания для систем ртуть-цинк и ртуть-свинец (см. таблицу). Расчет для первой системы проведен на основе экспериментальных данных [5], для второй на основе собствен- 
ных данных. Эксперименты проводились на тщательно обезжиренных наклонных $\left(30^{\circ} \leqslant \alpha \leqslant 90^{\circ}\right)$ пластинах поликристаллического свинца марки С-000 (общее содержание примесей меньше $4 \cdot 10^{-4} \%$ ) толщиной 1 мм и средним размером зерна 0,1 мм в среде $5 \%$-ного раствора трилона-Б $\left(\mathrm{C}_{10} \mathrm{H}_{14} \mathrm{O}_{8} \mathrm{~N}_{2} \mathrm{Na}_{2} \cdot 2 \mathrm{H}_{2} \mathrm{O}\right)$ при комнатной температуре (трилон-Б растворяет пленку окисла свинца).

Из таблицы видно, что экспериментальные значения показателей степени $n$ на всех интервалах параметра $x / x_{0}$ для обеих систем хорошо согласуются с расчетными. Среднее значение убыли удельной свободной поверхностной энергии в процессе растекания для системы ртуть-цинк

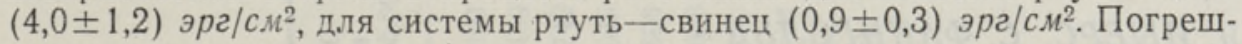
ность в оценке величины $\Delta \sigma$ определяется прежде всего погрешностью в нахождении коэффициента пропорциональности $A$ (относительная погрешность $\triangle A / A$ для рассматриваемых экспериментальных данных приведена для различных интервалов параметра $x / x_{0}$ в таблице).

Полученные значения $\Delta \sigma$ удовлетворительно согласуются с данными о кинетике растекания капли ртути с массой $m<20$ мг по поверхности горизонтально расположенных пластин цинка и свинца $[2,3]$. Это соответствие можно рассматривать как еще одно экспериментальное подтверждение применимости рассматриваемого метода определения движущей силы процесса растекания жидкости по поверхности твердого тела.

Автор приносит глубокую благодарность Е. Д. Шукину и Б. Д. Сумму за постановку задачи и обсуждение работы.

\section{Л ИТЕ РАТУРА}

1. Д е р я гин Б. В., ДАН СССР, 51, 357 (1946).

2. Р ауд Э. А., Сумм Б. Д., Щукин Е. Д., ДАН СССР, 205, 1134 (1972).

3. Шукин Е. Д., Горюнов Ю. В., ДеньщиковаГ. И., Перцов Н. В., С у м м Б. Д., Коллоидн. журн,, 25, 108 (1963).

4. Л а шко-А в акян С. В., Л а шко Н. Ф., Пайка металлов, М., 1959.

5. Дол жиков а В. Д., Сумм Б. Д., Горюн о В. В., В сб.: Физическая химия поверхностных явлений в расплавах, Киев, 1971, с. 194.

Институт физической химии Академин наук СССP

\section{Поступила в редакцию} 8/VIII 1972

\section{E. RAUD}

\section{VEDELIKU VALGUMIST MOODA TAHKE KEHA PINDA POHJUSTAVA JOU MAARAMISE MEETOD}

Töötati välja vedeliku valgumist mööda tahke keha pinda pōhjustava jōu määramise meetod. Leiti, et see jōud on arvuliselt vōrdne vaba pindenergia vähenemisega $(\Delta \sigma)$ valgumisprotsessi kestel. Meetod tugineb mööda tahket kaldplaati tōusva njutoonilise vedeliku peene kihi kohta koostatud Navje-Stoksi vörrandi lahendusele. Süsteemide $\mathrm{Hg}-\mathrm{Zn}$ ja $\mathrm{Hg}-\mathrm{Pb}$ kohta arvutatud $\Delta \sigma$ väärtused on heas vastavuses katseandmetega.

\section{E. RAUD}

\section{A METHOD OF CALCULATING THE MOVING FORCE OF THE WETTING PROCESS}

A method of calculating the moving force of the wetting process is developed. This force is numerically equal to the decrease of the free surface energy $(\Delta \sigma)$ during wetting. The method is based on the analysis and solution of the Navier-Stokes equation for the thin Newtonian liquid layer flowing along inclined and vertical solid plates. The calculated values $\Delta \sigma$ for systems $\mathrm{Hg}-\mathrm{Zn}$ and $\mathrm{Hg}-\mathrm{Pb}$ are in good agreement with experimental data. 